\title{
To Compare the Efficacy of Oral Midazolam and Triclofos Sodium as Oral Premedication in Children of Sawangi, Maharashtra, India
}

\author{
Aruna Vijay Chandak ${ }^{1}$, Deepjit Bhuyan², Krishnendu S. ${ }^{3}$, Vijay Chandak ${ }^{4}$ \\ 1,2,3,4 Department of Anaesthesia, Jawaharlal Nehru Medical College (JNMC), \\ Datta Meghe Institute of Medical Sciences (DU), Sawangi (Meghe), Wardha, Maharashtra, India.
}

\section{ABSTRACT}

\section{BACKGROUND}

The perioperative habits contemplate showing that kids are in more danger of encountering fierce sedative enlistment and unfriendly social sequelae. In paediatric sedation, decent premedication is fundamental to lessen tension and disturbance in youngsters. Parental partition and odd operating room environment as a rule bring about blustery acceptance while giving general sedation. Narcotic premedication is vital for making kids quiet and cooperative in a weird environment. Our study was done to compare the efficacy of midazolam $0.5 \mathrm{mg} / \mathrm{kg}$ and triclofos sodium $100 \mathrm{mg}$ / $\mathrm{kg}$ as oral premedications in children undergoing elective surgery.

\section{METHODS}

In this prospective randomised comparative study, sixty children posted for elective surgery were enrolled. The patients were randomly divided into midazolam group (Group M) and triclofos sodium group (Group T) of thirty each. Group M received oral midazolam $0.5 \mathrm{mg} / \mathrm{kg} 30 \mathrm{~min}$ before induction, and Group T received oral triclofos sodium $100 \mathrm{mg} / \mathrm{kg} 60 \mathrm{~min}$ before induction. All children were evaluated for level of sedation after premedication, behaviour at the time of separation from parents and at the time of mask placement for induction of anaesthesia.

\section{RESULTS}

Oral midazolam showed satisfactory sedation in children after premedication when compared to oral triclofos $(\mathrm{P}=0.003)$. Both the drugs had a successful separation from parents, and the children were very cooperative during induction. No adverse effects attributable to the premedicants were seen.

\section{CONCLUSIONS}

Oral midazolam is better than triclofos sodium as a sedative anxiolytic in the paediatric population.

\section{KEY WORDS}

Anaesthesia, Hypnotics and Sedatives, Midazolam, Paediatrics, Premedication, Triclofos sodium

\author{
Corresponding Author: \\ Dr. Deepjit Bhuyan, \\ Department of Anaesthesia, \\ Jawaharlal Nehru Medical College (JNMC), \\ Datta Meghe Institute of Medical \\ Sciences (DU), Sawangi (Meghe), \\ Wardha, Maharashtra, India. \\ E-mail:pooplibhuyan5@gmail.com
}

\section{DOI: 10.14260/jemds/2021/608}

How to Cite This Article:

Chandak AV, Bhuyan D, Krishnendu S, et al. To compare the efficacy of oral midazolam and triclofos sodium as oral premedication in children of Sawangi, Maharashtra, India. $J$ Evolution Med Dent Sci 2021;10(35):2975-2979, DOI: 10.14260/jemds/2021/608

Submission 25-03-2021,

Peer Review 29-05-2021,

Acceptance 05-06-2021,

Published 30-08-2021.

Copyright (C) 2021 Aruna Vijay Chandak et al. This is an open access article distributed under Creative Commons Attribution License [Attribution 4.0 International (CC $B Y 4.0)]$ 


\section{BACKGROUND}

Operation theatre surroundings, medical procedures and sedation cause pressure and nervousness which can prompt mental aggravations in kids. Besides, youngsters conceded to clinics are uprooted from their usual range of familiarity of home and family. In paediatric sedation, decent premedication is fundamental to lessen the tension and disturbance in youngsters. Parental partition and odd operating room environment as a rule bring about blustery acceptance while giving general sedation. Narcotic premedication is vital for making kids quiet and coemployable in a weird environment. Legitimate pre-operative arranging is important to limit the unfavourable mental impact in youngsters during the whole perioperative experience. Oral route is least traumatic and easily accepted. ${ }^{1}$

Midazolam is a brief-acting drug. It is a hypnotic agent and a good sedative drug with anxiolytic, muscle relaxant, and anticonvulsant properties. It has soothing, entrancing, and amnesic properties. It has a place with a class of medications called benzodiazepines. Midazolam is a special medication and this unique speciality makes it different from the other drugs of the same class because of its quick impact and a brief term of activity. Midazolam is accessible by various routes. The common routes of administration are oral, rectal, intranasal, intramuscular (IM), and intravenous (IV). Midazolam has been utilized and used in different biomedical applications, including dentistry, cardiovascular medical procedure, and endoscopic techniques as a presedative medicine, and as an assistant to nearby sedation. Midazolam is a brief-acting benzodiazepine central nervous system (CNS) depressant. Pharmacodynamic properties of midazolam and its metabolites, which are like those of other benzodiazepine drugs, incorporate calming, anxiolytic, amnestic, muscle relaxant, just as entrancing activities. Benzodiazepines improve the inhibitory activity of the amino acid neurotransmitter gamma-aminobutyric acid (GABA). Receptors for GABA are designated by numerous significant medications that influence GABA work and are ordinarily utilized in the treatment of nervousness problems, epilepsy, insomnia, spasticity, and forceful conduct.

Triclofos is a hypnotic. It is converted in the body into an active compound that works in the brain to induce sleepiness. It decreases the time taken to fall asleep and ssssincreases sleep duration. Chloral hydrate and triclofos initiate sedation adequately when given orally. They are processed to trichloroethanol which causes drug leftover impact and delayed sedation. Chloral hydrate tastes disagreeable and causes gastric bothering. Chloral hydrate is contraindicated in patients with liver sickness because the digestion will be deferred which can prompt metabolic acidosis, renal disappointment and hypotonia. In spite of the fact that triclofos is more attractive, it is slower to act and less strong than chloral hydrate.

The ideal premedicant ought to have a fast and dependable onset, negligible results, quick recuperation and ought to work with smooth acceptance of sedation. Oral midazolam satisfies a large number of the standards needed for an ideal premedicant. A dosage of $0.25-0.5 \mathrm{mg} / \mathrm{kg}$ of midazolam orally has been demonstrated to be effective in youngsters with fewer side effects. ${ }^{1,2}$
A settled type of chloral hydrate, triclofos sodium is a more seasoned non-narcotic, non-benzodiazepine oral sedative hypnotic drug utilized for paediatric sedation in a dose of $40-100 \mathrm{mg} / \mathrm{kg}$ for quite a long time. It is more acceptable than chloral hydrate. The oral arrangement is very much consumed and delivers entrancing for $6-8$ hours. $^{3}$

The study was intended to think about the impacts of midazolam and triclofos sodium when given as oral premedication in kids. The most essential goal was the soothing impact after premedication, and the auxiliary destinations were the anxiolytic impact at the hour of separation of kids from guardians and at the hour of mask application during sedation.

\section{METHODS}

After acquiring proper approval from the Institutional Ethics Committee, this prospective randomised comparative study was commenced. The study was conducted from July 2020 to December 2020. A well-informed written consent was taken from the parents and guardians of all children after explaining the nature of the study. The openepi.com software was used to find out the sample size. The sedation score of 4 attained by $93.33 \%$ of the participants (study by Radhika P. et al.) was taken into account, keeping the power at $80 \%$ and certainty interim at $95 \%$ (alpha error at 0.05 ), an example of 25 patients would be required to distinguish at least $20 \%$ contrast in the sedative effect between the two groups. 30 patients for each group were taken to make up for potential dropouts. In this study, sixty children of either gender participated. Each group was divided into thirty children. All of them belonged to the American Society of Anesthesiologists physical status I or II. The following children were excluded from the study: $<1$ year of age and with a body weight of more than $20 \mathrm{~kg}$, children with a difficult airway, mental retardation, central nervous system disorders, on anticonvulsants, or sedative medications and those who required additional sedation. Children who had taken part in the study belonged to the age group of 1-8 years and went for elective surgery.

Pre-operative assessment was performed and nil by mouth orders was according to the standard convention. Patients were randomly assigned to the midazolam bunch (Group M) and triclofos sodium bunch (Group T). Oral midazolam was administered at a dose of $0.5 \mathrm{mg} / \mathrm{kg} 30 \mathrm{~min}$ before the anticipated time of induction of anaesthesia in group M. Syrup triclofos sodium was given in a portion of $100 \mathrm{mg} / \mathrm{kg}$, in group T, $60 \mathrm{~min}$ before the anticipated time of induction of anaesthesia. The children were made to relax along with their parents in an undisturbed area after the medications were given. The level of sedation post premedication, simplicity of partition from guardians and the conduct during mask application were the boundaries that were surveyed. The level of sedation was graded by 5 point score $(1$ = asleep not readily arousable, 2 = asleep responds slowly to gentle stimulation, 3 = drowsy readily responds, $4=$ awake calm and quiet, $5=$ awake active). ${ }^{4}$ The behaviour at the time of separation from parents was assessed when the child was separated from its parents to shift to the operating room using the separation score $(1=$ excellent, happily 
separated, 2 = good, separated without crying, 3 = fair, separated with crying, $4=$ poor, need for restraint). A score of $\leq 2$ was considered successful while a score $>2$ was considered unsuccessful. Face mask placement behaviour was assessed by co-operation score $(1=$ co-operative, $2=$ mildly resistant, 3 = resists placement of mask).

Side-effects of oral premedicants such as nausea, vomiting, hiccups, airway obstruction, restlessness, or slurring of speech were noted after the drug administration and in the recovery period. ${ }^{4}$

\section{Statistical Analysis}

It was performed using the SPSS 16.0 (Statistical Package for the Social Science for windows; Version 16.0, SPSS Inc., Chicago, USA). Results were analysed using Student's $t$-test for parametric data and Mann-Whitney U-test for nonparametric data. Significance level was set at $\mathrm{P}<0.05$.

\section{RESULTS}

After premedication, $96.66 \%$ of kids of the midazolam group (M) were adequately sedated (sedation score 4) compared to $63.33 \%$ in the triclofos group (T) [Figure1]. The differences in sedation between the two groups were found to be statistically significant with $\mathrm{P}=0.002$ [Table1]. None of the kids had oxygen saturation levels below $98 \%$.

\begin{tabular}{|cccc|}
\hline \multicolumn{2}{|c|}{ Sedation Score } & Group T (N = 30) & (\%) Group M (N = 30) (\%) P - Value \\
1 & 0 & 0 & \\
2 & $0(33.33)$ & $1(3.33)$ & $0.003^{*}$ \\
3 & $1(3.33)$ & 0 & \\
4 & $19(63.33)$ & $29(96.66)$ \\
5 & 0 & 0 \\
\hline Table 1. Comparison of Sedation Scores Post - Premedication \\
\hline *- significant
\end{tabular}

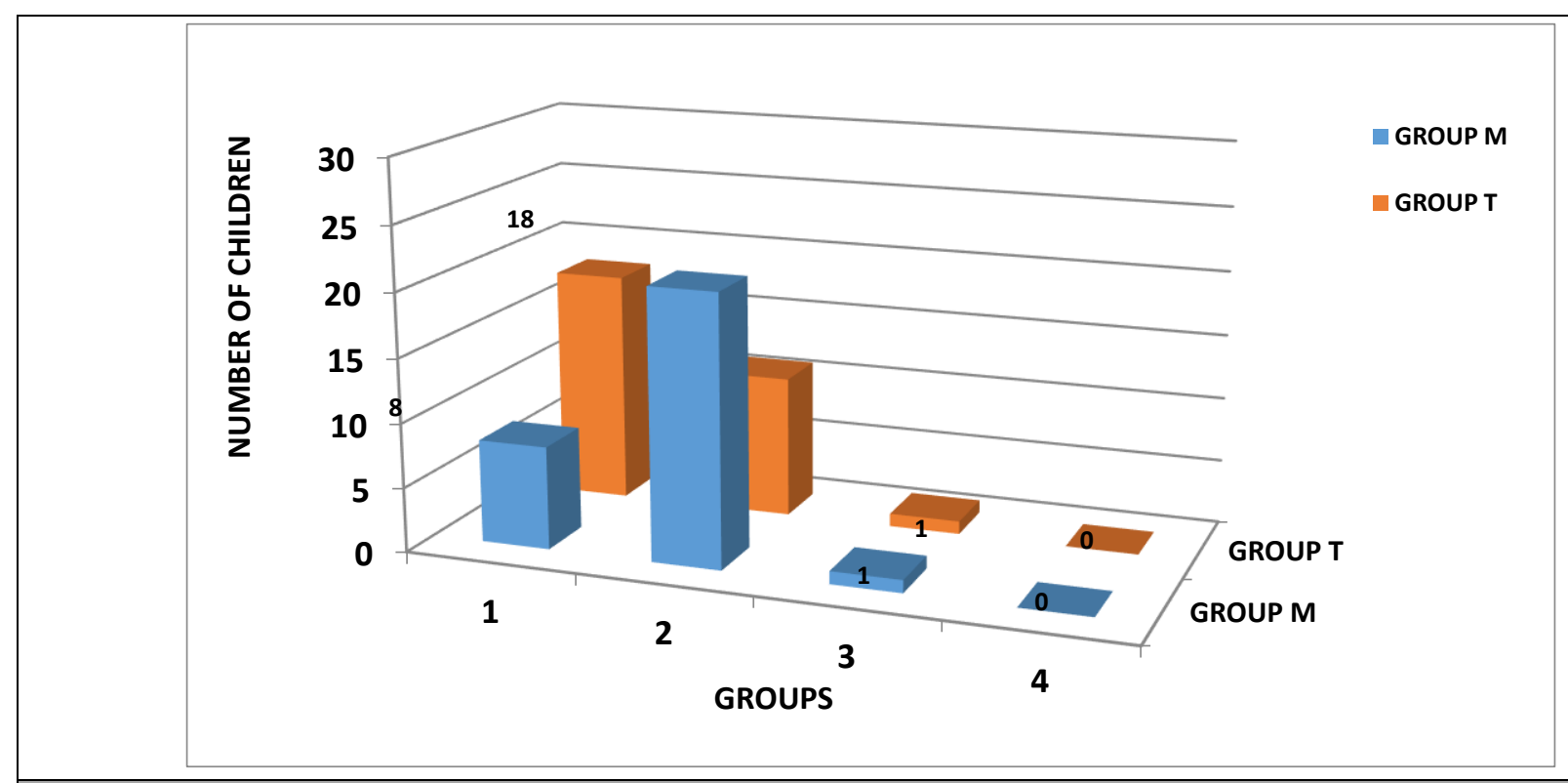

Figure 1. Level of Sedation after Premedication (Assessed after $30 \mathrm{~min}$ in Midazolam Group and after 60 min in Triclofos Group) $1=$ Asleep Not Readily Arousable, 2 = Asleep Responds Slowly to Gentle Stimulation, 3 = Drowsy Readily Responds, $4=$ Awake Calm and Quiet, 5 = Awake Active

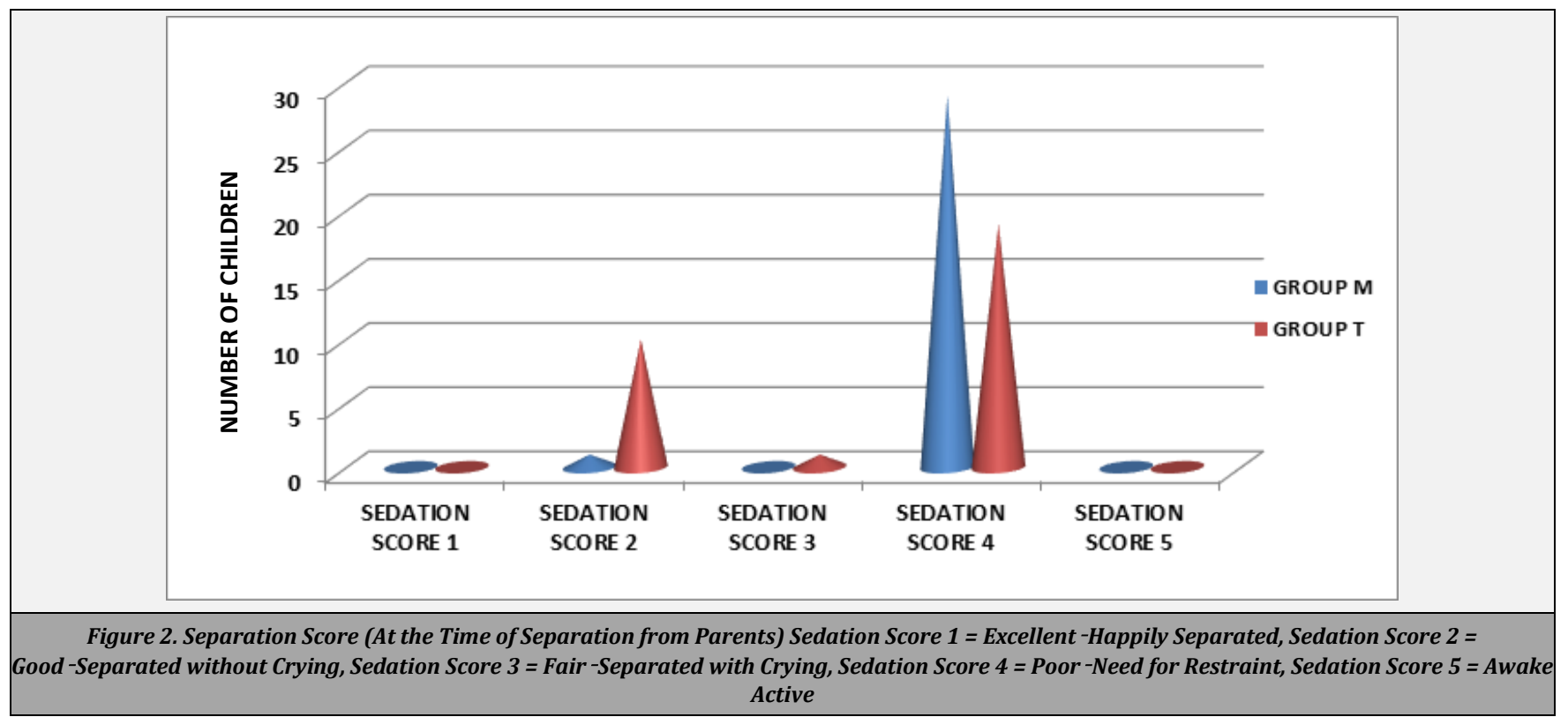


The separation score of both the groups had an equal number of children [Figure 2 and Table - 2]. Successful separation (scores 1 and 2) was clubbed together as successful.

\begin{tabular}{|cccc|}
\hline Separation Score & $\begin{array}{c}\text { Group T (N = 30) } \\
(\%)\end{array}$ & $\begin{array}{c}\text { Group M (N = 30) (\%) } \\
\text { Value } \\
\text { Valu }\end{array}$ \\
\hline 2 & $18(60)$ & $8(26.66)$ & \\
3 & $11(36.66)$ & $21(70)$ & 0.06 \\
4 & $1(3.33)$ & $1(3.33)$ & \\
\hline \multicolumn{4}{|c}{ Table2. Comparison of Separation Score } \\
\hline
\end{tabular}

When mask application was performed $86.6 \%$ of children belonging to the midazolam group (M) were co-operative compared to $93.3 \%$ of the triclofos group (T). The difference found between the two groups was statistically not significant with a $\mathrm{P}=0.278$ [Table 3].

\begin{tabular}{|cccc|}
\hline Score & Group M (N = 30) (\%) & Group T (N = 30) (\%) & P - Value \\
1 & $26(86.66)$ & $28(93.33)$ & \\
2 & $4(13.33)$ & $2(6.66)$ & 0.278 \\
3 & 0 & 0 & \\
\hline \multicolumn{4}{|c|}{ Table 3. Comparison of Cooperation Score (Face Mask Acceptance by } \\
the Child)
\end{tabular}

No adverse effects attributable to premedicants were seen in both the groups.

\section{DISCUSSION}

In paediatric sedation, a decent premedicant is needed to limit the mental pressure and control a troubled youngster. It should make the kid quiet and calm during enlistment of sedation and ought to have no antagonistic cardiovascular or respiratory ventilatory impacts. It has to be solid in the onset of activity with insignificant results, ought to give quick recuperation and get back to readiness postoperatively allowing simple release from the recovery room.

Perioperative period is normally a horrendous encounter for youngsters going through a medical procedure. In kids, premedication is used to guarantee a quiet co-usable kid without creating profound sedation and respiratory wretchedness. Anxiolysis or sedation is the point of presentday premedication to get a quiet and cooperative kid. There are different courses for premedication organization yet oral course stays the course of decision for youngsters since it is not difficult to regulate and least threatening to kids. Along these lines in this examination, all medications were given orally as the sole specialist. The time-stretch among premedication and detachment from guardians ought to give sufficient sedation and anxiolysis at the hour of separation from guardians and the hour of mask placement for acceptance of sedation in the working room.

In the current study, midazolam syrup was given in the dose of $0.5 \mathrm{mg} / \mathrm{kg}$. Studies by Kolathu PR et al. ${ }^{1}$ Choudhary S et al. $^{2}$ Geetha $\mathrm{L}^{3}$ showed that the dose of $0.5 \mathrm{mg} / \mathrm{kg}$ of midazolam orally has proven to be efficacious in children with fewer side effects. Hence a dose of $0.5 \mathrm{mg} / \mathrm{kg}$ was chosen for midazolam. Triclofos syrup was used in the dose of 75 mg / kg Radhika KP, Sreejit MS, Ramadas KT used triclofos $100 \mathrm{mg} / \mathrm{kg}$ and concluded that this was the safe dose with fewer side effects, hence the dose of $100 \mathrm{mg} / \mathrm{kg}$ was chosen for triclofos. Midazolam is rapidly absorbed in the gastrointestinal tract and produces its peak effect in 30 mins. ${ }^{5}$ Triclofos oral solution is well-absorbed and shows efficacy within 30 - 40 minutes. ${ }^{5,4}$ Choudhary S et al. ${ }^{2}$ and Geetha L et al. ${ }^{3}$ reported that the maximum percentage of patients achieved excellent sedation scores at $60 \mathrm{~min}$. Hence, the time for assessment of sedation for triclofos was selected as 60 minutes ${ }^{2}$ and for midazolam as 30 minutes.

On comparing the level of sedation in both the groups, it was observed that in group - M, $96.66 \%$ of patients had achieved a score of 4 (awake calm and quiet) while only $63.33 \%$ of patients in group - $\mathrm{T}$ achieved a score of 4 which is shown in Table 1 . Our study was comparable to studies carried out by Kolathu PR et al. ${ }^{1}$ and Geetha L et al. ${ }^{3}$ Jose MR et al. $^{6}$ observed that in the midazolam group $88 \%$ of patients had a sedation score of 2 whereas in the triclofos group $84 \%$ had sedation score of 4 . In our study, both groups had an equal number of children [Figure 2 and Table 2] with successful separation (scores 1 and 2 clubbed together as successful). Kolathu PR et al. ${ }^{1}$ Choudhary $S$ et al. ${ }^{2}$ Jose MR et al. ${ }^{6}$ Geetha et al. ${ }^{3}$ have observed no difference in separation score. When behaviour during mask acceptance was compared in both the groups, it was seen that a significantly higher proportion of patients in group - $\mathrm{T}$ achieved a score of $1=$ cooperative, accepts mask easily (93.33\%) as compared to group - M wherein only $86.66 \%$ patients achieved score 1 (Table 3). This study correlates with the studies carried out by Chaudhary S et al. ${ }^{2}$ Parmeshwari A et al. ${ }^{7}$ None of the patients in any of the groups had side effects like nausea, vomiting, rigours, hypotension, bradycardia etc. which was similar to the studies carried out by Kolathu PR et al. ${ }^{1}$ Chaudhary $\mathrm{S}^{2}$, Jose MR et al. ${ }^{6}$ Parmeshwari A et al. ${ }^{7}$ and Geetha L. ${ }^{3}$

\section{CONCLUSIONS}

Medical clinic affirmation, sedation and medical procedure are distressing encounters for kids which may prompt mental injury and character changes. The expanding utilization of day-care as a medical procedure, the aversion of parental division and the utilization of narcotic premedication may diminish the pressure of hospitalization in kids and the danger of unfavourable mental spin-off. Kids premedicated with oral midazolam were sufficiently calmed in contrast to oral triclofos sodium. This adds to the well-being edge after premedication with oral midazolam. The nature of acceptance of sedation was like that of triclofos sodium. Oral midazolam is better than oral triclofos sodium as calming anxiolytic in the paediatric populace.

Data sharing statement provided by the authors is available with the full text of this article at jemds.com.

Financial or other competing interests: None.

Disclosure forms provided by the authors are available with the full text of this article at jemds.com. 


\section{REFERENCES}

[1] Kolathu PR, Melveetil SS, Konnanath TR. Efficacy of midazolam as oral premedication in children in comparison to triclofos sodium. Indian J Anaesth 2016;60(6):415-19.

[2] Chaudhary S, Jindal R, Girotra G, et al. Is midazolam superior to triclofos and hydroxyzine as premedicant in children? Journal of Anaesthesiology Clinical Pharmacology 2014;30(1);53-8.

[3] Geetha L, Sunitha KS. Efficacy of oral triclofos compared with oral midazolam as premedication in paediatric age group. Pediatric Anesthesia and Critical Care Journal 2018;6(1):31-7.
[4] Bhatnagar S, Das UM, Bhatnagar G. Comparison of oral midazolam with oral tramadol, triclofos and zolpidem in the sedation of pediatric dental patients: an in vivo study. Journal of Indian Society of Pedodontics and Preventive Dentistry 2012;30(2):109-14.

[5] Pandit UA, Collier PJ, Malviya S, et al. Oral transmucosal midazolam premedication for preschool children. Can J Anaesth 2001;48(2):191-5.

[6] Jose MR, Vinod S. Premedicant in children: efficacy of oral Midazolam Vs. Triclofos. International Journal of Recent Trends in Science and Technology 2015;13(3):625-9.

[7] Parameswari A, Maheedar G, Vakamudi M. Sedative and anxiolytic effects of midazolam and triclofos oral premedication in children undergoing elective surgery: a comparison. J Anaesth Clin Pharmacol 2010;26(3):340-4. 